\title{
226. On Realization of the Discrete Series for Semisimple Lie Groups
}

\author{
By Ryoshi Hotta \\ (Comm. by Kunihiko KodaIra, M. J. A., Nov. 12, 1970)
}

This note is an announcement of a result, which says, briefly, that most of the discrete series for a semisimple Lie group are realized as certain eigenspaces of the Casimir operator on the symmetric space (Theorem 2). This construction is in some sense a generalization of the methods adopted in [1], [2], [9] for special groups and in [5] for the groups of hermitian type. Also, [6] indicates the above method of realization. Further, as for alternative methods to realize most of the discrete series, we refer to the recent works [5], [8]. Our technique used here depends heavily on that of [5]. A detailed exposition with full proofs will appear elsewhere.

1. Let $G$ be a connected non-compact semisimple Lie group with a compact Cartan subgroup. We assume, for convenience, that $G$ has a faithful finite dimensional representation and its complexification $G^{C}$ is simply connected. Fix a maximal compact subgroup $K$ of $G$ and a Cartan subgroup $H$ contained in $K$. We denote by $\mathfrak{g}, \mathfrak{f}$ and $\mathfrak{h}$ the Lie algebras corresponding to $G, K$ and $H$ respectively. For complexifications $\mathfrak{g}^{C}, \mathfrak{l}^{C}, \mathfrak{h}^{C}$ of $\mathfrak{g}, \mathfrak{f}, \mathfrak{h}$, we denote by $\Delta$ the root system of $\left(\mathfrak{g}^{C}, \mathfrak{h}^{C}\right)$, and by $W_{G}$ the Weyl group of $\left(\mathfrak{f}^{C}, \mathfrak{h}^{C}\right)$. Taking a positive root system $P$ of $\Delta$ fixed once for all, $P_{k}$ (resp. $P_{n}$ ) denotes the set of a positive compact (resp. non-compact) roots. Let $L$ be the character group of $H, L^{\prime}$ the set of regular elements in $L$. Introducing an inner product (,) on $L$ induced by the Killing form, we put $\varepsilon(\lambda)=\operatorname{sign} \prod_{\alpha \in P}(\lambda, \alpha)$ for $\lambda \in L^{\prime}$, and $\varepsilon(\lambda)=0$ for $\lambda \in L-L^{\prime}$. We also put $\varepsilon_{k}(\lambda)=\operatorname{sign} \prod_{\alpha \in P_{k}}(\lambda, \alpha)$ if $\lambda \in L$ is $\mathfrak{f}^{C}$-regular, and $\varepsilon_{k}(\lambda)=0$ if $\lambda$ is $\mathfrak{f}^{C}$-singular. For discrete series, the following fact is known by Harish-Chandra [3]. Let $\mathcal{E}_{d}$ be the discrete series for $G$. For $\lambda \in L^{\prime}$, there then exists a unique element $\omega(\lambda) \in \mathcal{E}_{d}$, and the map $L^{\prime} \ni \lambda \mapsto \omega(\lambda) \in \mathcal{E}_{d}$ is surjective, while $\omega(\lambda)=\omega\left(\lambda^{\prime}\right)$ if and only if there exists $w \in W_{G}$ such that $w \lambda=\lambda^{\prime}$. We shall denote by $\Theta_{\omega(\lambda)}$ the character of $\omega(\lambda)$.

For a finite subset $A$ of $L$, we shall denote by $|A|$ its cardinal number and put $\langle A\rangle=\sum_{\alpha \in A} \alpha$. Put $\rho=\langle P\rangle / 2, \rho_{k}=\left\langle P_{k}\right\rangle / 2$ and $\rho_{n}=\rho-\rho_{k}$. If $\varepsilon_{k}\left(\lambda+\rho_{k}\right) \neq 0$ for $\lambda \in L$, there exists a unique $w \in W_{G}$ such that $w\left(\lambda+\rho_{k}\right)-\rho_{k}$ is $k^{C}$-dominant. We then denote by [ $\left.\lambda\right]$ the equivalence class to which belongs an irreducible $K$-module with highest weight 
$w\left(\lambda+\rho_{k}\right)-\rho_{k}$. For the sake of notational convenience, we put $[\lambda]=0$ if $\varepsilon_{k}\left(\lambda+\rho_{k}\right)=0$. We shall denote by $\chi(\lambda)$ the character of [ $\left.\lambda\right]$.

2. For a finite dimensional unitary $K$-module $V$, we denote by $C V$ the homogeneous vector bundle over $G / K$ associated to $V$, whose fiber has an invariant hermitian metric. Throughout this note, for a $K$ module the corresponding script letter denotes the homogeneous vector bundle associated to the $K$-module given. Let $L_{2}(C V)$ (resp. $\left.C^{\infty}(C V)\right)$ be a space of all square-integrable (resp. differentiable) sections of $C V$, which is naturally regarded as the space consisting of all $V$-valued square-integrable (resp. differentiable) functions $f$ satisfying $f(g k)$ $=k^{-1} f(g)$ for $k \in K, g \in G$. Now assume that there are given two $K$ modules $V, W$. For a $G$-invariant linear differential operator $D: C^{\infty}(C V)$ $\rightarrow C^{\infty}(\mathscr{W})$, the maximal extension $D: L_{2}(C V) \rightarrow L_{2}(\mathscr{W})$ means the closed linear operator whose domain consists of $f \in L_{2}(C V)$ such that $D f \in L_{2}(\mathscr{W})$ in the sense of distributions. We shall hereafter consider differential operators on square-integrable sections in this sense. Let $D^{*}: L_{2}(\mathscr{W})$ $\rightarrow L_{2}(C V)$ be the maximal extension of the formal adjoint operator for $D$. We then have the unitary representations of $G$ on the Hilbert spaces $\operatorname{Ker} D$ and $\operatorname{Ker} D^{*}$. Let $(\operatorname{Ker} D)_{d}\left(\operatorname{resp} .\left(\operatorname{Ker} D^{*}\right)_{d}\right)$ be the smallest closed invariant subspace which contains every irreducible closed invariant subspace of $\operatorname{Ker} D\left(\operatorname{resp} . \operatorname{Ker} D^{*}\right)$. Denote by $\pi_{V}\left(\operatorname{resp} . \pi_{W}\right)$ the representation on the space $(\operatorname{Ker} D)_{d}\left(\operatorname{resp} .\left(\operatorname{Ker} . D^{*}\right)_{d}\right)$. It is then shown that the operator $\pi_{V}(\varphi)=\int_{G} \varphi(g) \pi_{V}(g) d g$ is of trace class for a compactly supported $C^{\infty}$-function $\varphi$ on $G$, and so defines an invariant distribution Trace $\pi_{V}$ on $G$ (the same holds also for $\pi_{W}$ ). The following theorem can be proved by a similar method to the one in [5].

Theorem 1. Under the above situation, assume that $D$ is at most a first order operator, and denote by $\chi_{V}, \chi_{W}$ the characters of $V, W$. Suppose that

$$
\chi_{V}-\chi_{W}=\varepsilon_{k}(\lambda+\rho) \sum_{Q \subset P_{n}}(-1)^{\left|Q_{\lambda}\right|} \chi(\lambda+\langle Q\rangle)
$$

for some $\lambda \in L$ such that $\varepsilon(\lambda+\rho) \neq 0$. Then

Trace $\pi_{V}-$ Trace $\pi_{W}=(-1)^{\left|Q_{\lambda}\right|} \Theta_{\omega_{\alpha(\lambda+\rho)}}$

where $Q_{\lambda}=\left\{\beta \in P_{n} ;(\lambda+\rho, \beta)>0\right\}$.

Corollary. For $\lambda \in L$, take such $K$-modules $V, W$ as [V] $=\oplus[\lambda+\langle Q\rangle]$ where the summation runs over every $Q \subset P_{n}$ such that $\varepsilon_{k}(\lambda+\rho) \varepsilon_{k}\left(\lambda+\rho_{k}+\langle Q\rangle\right)=(-1)^{|Q|}$, and as $[W]=\oplus[\lambda+\langle Q\rangle]$ where the summation runs over every $Q \subset P_{n}$ such that $\varepsilon_{k}(\lambda+\rho) \varepsilon_{k}\left(\lambda+\rho_{k}+\langle Q\rangle\right)$ $=(-1)^{|Q|+1}$. Then for any first order operator $D$, the formula in Theorem 1 holds. Here, [V], $[W]$ denote the equivalence classes to which $V, W$ belong.

3. Let $V_{\lambda+\langle Q\rangle}$ be an irreducible $K$-module belonging to $[\lambda+\langle Q\rangle]$ for $\lambda \in L, Q \subset P_{n}$, when $\varepsilon_{k}\left(\lambda+\rho_{k}+\langle Q\rangle\right) \neq 0$, and denote by $w_{\lambda+\langle Q\rangle}$ the 
unique element of $W_{G}$ such that $w_{\lambda+\langle Q\rangle}\left(\lambda+\rho_{k}+\langle Q\rangle\right)$ is $k^{C}$-dominant. Let $\Omega$ be the Casimir operator of $G$. Then the action of $\Omega$ on $L_{2}(G)$ as a left invariant differential operator defines the action $\nu(\Omega)$ on $L_{2}\left(C \nu_{\lambda+\langle Q\rangle}\right)$ because $\Omega$ belongs to the center of the universal enveloping algebra of $g^{C}$. Put $H_{\lambda}^{Q}=\left\{f \in L_{2}\left(C V_{\lambda+\langle Q\rangle}\right) ; \nu(\Omega) f=(\lambda+2 \rho, \lambda) f\right\}$. For $w \in W_{G}$, we put $A_{\lambda}(w, Q)=\left(\rho-\langle Q\rangle, 2\left\langle Q_{\lambda}\right\rangle-\langle Q\rangle-\rho\right) / 2+\left(\rho_{k}, \rho_{n}-\left\langle Q_{\lambda}\right\rangle-w\left(\rho_{n}-\left\langle Q_{\lambda}\right\rangle\right)\right)$ $+(\rho, \rho) / 2$. We then have the following lemma in a similar way to the one in $[6]$.

Lemma. If $|(\lambda+\rho, \beta)|>A_{\lambda}\left(w_{\lambda+\langle Q\rangle}, Q\right)$ for every $\beta \in P_{n}$, then $H_{\lambda}^{Q}=0$ if $Q \neq Q_{\lambda}$.

In [4], we obtained an elliptic complex $C V_{\lambda}^{*}$ whose first term is the homogeneous vector bundle associated to an irreducible $K$-module $V_{\lambda}$ with lowest weight $\lambda+2 \rho_{k}$ (the $(\#)$-complex for $\lambda$ under an admissible linear order of $\Delta$ in terminology of [4]). One can define the squareintegrable "cohomology" space $H_{2}^{q}\left(C V_{\lambda}^{*}\right)$ for this elliptic complex. The following proposition is shown by Theorem 1 and the above Lemma.

Proposition. There exists a non-negative constant a such that the following holds. If $|(\lambda+\rho, \alpha)|>a$ for every $\alpha \in P$, then $H_{2}^{q_{2}}\left(C V_{\lambda}^{*}\right) \neq 0$ and the irreducible unitary representation of $G$ with character $\Theta_{\omega(\lambda+\rho)}$ is realized as a closed subspace of $H_{2}^{q_{\lambda}}\left(C V_{\lambda}^{*}\right)$ for $q_{\lambda}=\left|Q_{\lambda}\right|$.

4. For $\Lambda \in L^{\prime}$, choose a positive root system such as $P=\{\alpha \in \Delta$; $(\Lambda, \alpha)<0\}$ and fix the linear order on $\Delta$ induced by $P$. Put $\lambda=\Lambda-\rho$. Then $-\left(\lambda+2 \rho_{k}\right)$ is $\mathfrak{f}^{C}$-dominant with respect to this linear order. Let $V_{\lambda}$ be the irreducible $K$-module with lowest weight $\lambda+2 \rho_{k}$, and put $A(w, Q)=(\langle Q\rangle,\langle Q\rangle) / 2+\left(\rho_{k}, \rho_{n}-w \rho_{n}\right)$ and $b=\max _{w \in W_{G}, Q \subset P_{n}} A(w, Q)$. The next theorem follows from Corollary to Theorem 1 and Lemma in 3.

Theorem 2. If $|(\lambda+\rho, \beta)|>b$ for every $\beta \in P_{n}$, then the Hilbert space

$$
\mathscr{S}_{2}=\left\{f \in L_{2}\left(C \nu_{\lambda}\right) ; \nu(\Omega) f=(\lambda+2 \rho, \lambda) f\right\}
$$

gives an irreducible unitary representation belonging to the discrete series for $G$, whose character is $\Theta_{\omega(\lambda+\rho)}$.

Remark. In view of Harish-Chandra's result cited in 1, we see that "most" of the discrete series are realized in this procedure. This construction is partially a generalization of the method in [9] for the de Sitter group and an answer to the proposal in [6]. Further, when $(G, K)$ is a symmetric pair of hermitian type and that all elements in $P_{n}$ are totally positive, Theorem 2 is included in Proposition 9.1 in [5].

5. As for a relation with another realization of the discrete series, we shall refer to the one by means of Schmid's operator (see [4], [7]). For $\Lambda \in L^{\prime}$, we choose $P$ and define $\lambda, V_{\lambda}$ as in 4 . Put $c^{\sharp}=\mid \min _{\alpha \in P_{k}, Q \subset P_{n}}$ $\left(\rho_{n}-\langle Q\rangle, \alpha\right) \mid$. Then $\lambda$ satisfies the condition (\#) in terminology of [4] 
if $|(\lambda+\rho, \alpha)| \geqslant c^{\#}$ for every $\alpha \in P_{k}$. Hence for a $K$-module $V_{\lambda}^{1}$ whose irreducible components consist of $[\lambda+\beta]$ where $\beta$ runs over the elements on $P_{n}$, we have an elliptic first order operator

$$
\mathscr{D}: L_{2}\left(C V_{2}\right) \rightarrow L_{2}\left(C V_{\lambda}^{1}\right)
$$

(see [4], [7]). We denote by $\mathscr{H}_{\lambda}$ the null space of $\mathscr{D}$. Put $c^{\prime}=\mid \min _{\alpha \in P_{k}, \beta \in P_{n}}$ $\left(\rho_{k}-\rho_{n}-\beta, \alpha\right) \mid$. It is then easily seen that the multiplicity of [ $\left.\lambda\right]$ in $\mathcal{H}_{\lambda}$ is at most one, if $|(\lambda+\rho, \alpha)|>\max \left(c^{\sharp}, c^{\prime}\right)$ for every $\alpha \in P_{k}$, from Theorem 6.2 in [4] (see also [7]). Taking the unique element $w_{0} \in W_{G}$ such that $w_{0} P_{k}=-P_{k}$, we put $c^{\prime \prime}=\max _{Q \subset P_{n}} A\left(w_{0}, Q\right)$, and $c=\max \left(c^{\sharp}, c^{\prime}, c^{\prime \prime}\right)$. Combining the above fact with Theorem 1 and Lemma in 3, one can complete a proof of the following theorem.

Theorem $3^{*)}$. If $|(\lambda+\rho, \alpha)|>c$ for every $\alpha \in \Delta$, then $\mathcal{H}_{\lambda}$ gives an irreducible unitary representation with character $\Theta_{\omega(\lambda+\rho)}$.

Remark. Under the condition of Theorem 3, we see that $\mathcal{H}_{\lambda}$ is contained in $\mathfrak{S}_{\lambda}$. Moreover, we can show that $\mathfrak{S C}_{\mathcal{C}}^{\lambda}$ is irreducible, which implies that $\mathfrak{S}_{\lambda}=\mathcal{H}_{\lambda}$. Therefore, under this condition, the two procedures to realize the discrete series are equivalent.

\section{References}

[1] V. Bargmann: Irreducible unitary representations of the Lorentz group. Ann. of Math., 48, 568-640 (1947).

[2] Harish-Chandra: Representations of semisimple Lie groups. V. Amer. J. Math., 78, 1-41 (1956).

[3] - : Discrete series for semisimple Lie groups. II. Acta Math., 116, 1-111 (1966).

[4] R. Hotta: Elliptic complexes on certain homogeneous spaces. Osaka J. Math., 7, 117-160 (1970).

[5] M. S. Narasimhan and K. Okamoto: An analogue of the Borel-Weil-Bott theorem for hermitian symmetric pairs of non-compact type. Ann. of Math., 91, 486-511 (1970).

[6] K. Okamoto: On induced representations. Osaka J. Math., 4, 85-94 (1967).

[7] W. Schmid: Homogeneous complex manifolds and representations of semisimple Lie groups. Thesis, Proc. Nat. Acad. Sci. U.S.A., 59, 56-59 (1968).

[8] — : On a conjecture of Langlands. to appear.

[9] R. Takahashi: Sur les représentations unitaires des groupes de Lorentz généralisés. Bull. Soc. Math. France, 91, 289-433 (1963).

*) The fact in Theorem 3 was communicated in the letter from Prof. Schmid without a proof. 